\title{
Percepção dos Idosos em Relação a Vida Sexual e as Infecções Sexualmente Transmissíveis na Terceira Idade
}

\author{
Perception of the Elderly in Relation to the Sexual Life and the \\ Sexually Transmitted Infections
}

\author{
Laís Carolini Theis ${ }^{1}$ \\ Diandra Leite Gouvêa ${ }^{2}$
}

\section{RESUMO}

Objetivo: conhecer a percepção dos idosos em relação à vida sexual na terceira idade e às infecções sexualmente transmissíveis. Metodologia: estudo qualitativo com abordagem descritiva, realizado em um município da Região do Médio Vale do Itajaí em Santa Catarina. A coleta de dados foi realizada em uma instituição pública, que desenvolve atividades voltadas ao público idoso. Foram entrevistados dez sujeitos, com idade superior a 60 anos, por meio de uma entrevista aberta. Para análise dos dados, foram estabelecidas categorias através da técnica de análise de conteúdo. Resultados: a partir da análise das falas dos entrevistados, emergiram três categorias de análise, sendo elas: sexualidade na terceira idade: os aspectos físicos e emocionais sob o olhar dos idosos; a percepção dos idosos sobre as infecções sexualmente transmissíveis; a visão do idoso e o seu entendimento sobre a prevenção de infecções sexualmente transmissíveis. Conclusão: os resultados evidenciaram que os idosos possuem conhecimento acerca das infecções sexualmente transmissíveis e sua forma de prevenção. Porém, em sua maioria não realizam a prevenção por confiar no parceiro ou por não considerar necessário na sua condição atual.

DESCRITORES: Saúde do Idoso, Comportamento Sexual, Doença Sexualmente Transmissível.

\begin{abstract}
Objective: to know the perception of the elderly in relation to the sexual life in the third age and to the sexually transmissible infections. Methodology: qualitative study with a descriptive approach, carried out in a municipality of the Region of the Middle Vale do Itajaí in Santa Catarina. Data collection was performed in a public institution, which develops activities aimed at the elderly public. Ten subjects, aged over 60 years, were interviewed through an open interview. For data analysis, categories were established through the technique of content analysis. Results: from the analysis of the interviewees' speeches, three categories of analysis emerged: sexuality in the third age: the physical and emotional aspects under the eyes of the elderly; the perception of the elderly about sexually transmitted infections; the view of the elderly and their understanding about sexually transmitted infections. Conclusion: the results showed that the elderly have knowledge about sexually transmitted infections and their form of prevention. However, most do not carry out prevention by relying on the partner, or by not considering it necessary in their current condition.
\end{abstract}

DESCRIPTORS: Elderly Health, Sexual Behavior, Sexually transmitted disease.

1 - Doutoranda do Programa de Pós-Graduação em Odontologia, ênfase multidisciplinar em Saúde Coletiva. Pontifícia Universidade Católica do Paraná (PUCPR). Curitiba, Paraná, Brasil.

2 - Acadêmica de Enfermagem da Faculdade Metropolitana de Blumenau (FAMEBLU) - Uniasselvi. Blumenau, Santa Catarina, Brasil. 
$\mathrm{O}$ processo de envelhecimento ocorre sem idade definida, dependendo da disposição em relação à qualidade de vida, mas pode ser considerado um processo de alteração caracterizado fisiologicamente por alterações físicas, psicológicas, sociais e ambientais, ocorrendo de forma diferente, dependendo das situações econômicas e sociais do indivíduo. A terceira idade, em países em desenvolvimento, é definida por indivíduos com idade igual ou superior a 60 anos de idade, enquanto nos países desenvolvidos a partir dos 65 anos $^{1}$.

As infecções sexualmente transmissíveis (IST), causadas por múltiplas bactérias, fungos e vírus, através do ato sexual desprotegido, vêm aumentando na terceira idade, gerando impacto na relação familiar e social, sendo notório o aumento desse número em idosos, definido como um fenômeno global, que traz como implicação a necessidade de adequação dos serviços de saúde para atender as necessidades desses clientes ${ }^{2}$.

Atualmente, têm-se percebido mudanças no curso epidemiológico de Síndrome da Imunodeficiência Adquirida (AIDS) e, uma delas, é o aumento do número de casos entre idosos. Apesar disso, as informações quanto à doença, mostram que esses cidadãos ainda estão invisíveis no que diz respeito às políticas públicas de prevenção. Sendo considerada uma das principais IST que acometem os idosos por falta de medidas preventivas ${ }^{3}$.

Devido ao contexto histórico mistificado acerca da pessoa idosa e a sexualidade, torna esses indivíduos cada vez mais expostos à situação de vulnerabilidade. Além disso, evidencia-se uma dificuldade em diagnosticar precocemente o problema, devido a falta de profissionais capacitados a realizar o acolhimento e a escuta qualificada e, também, a baixa procura dos idosos aos serviços de saúde buscando informações acerca do tema ${ }^{4}$.

Diante da complexidade da temática esse estudo objetivou conhecer a percepção dos idosos em relação à vida sexual na terceira idade e as infecções sexualmente transmissíveis.

\section{Metodologia}

Trata-se de um estudo qualitativo com abordagem descritiva. O estudo foi realizado em um município da Região do Médio Vale do Itajaí, no Estado de Santa Catarina, em uma instituição pública, com programas voltados às pessoas idosas. Os critérios de inclusão foram: estar cadastrado no programa disponibilizado pela referida instituição, idade igual ou superior a 60 anos e que aceitaram participar do estudo mediante assinatura do Termo de Consentimento Livre e Esclarecido. Foram realizadas entrevistas semiestruturadas com perguntas abertas, por meio de um instrumento elaborado pelas autoras. A amostra foi composta de dez indivíduos, sendo interrompida a coleta devido esgotamento do tema.

Os participantes foram identificados de acordo com a inicial do gênero - homens em "H" e mulheres por "M" - e numeradas conforme ordem de entrevistas. As entrevistas foram gravadas e transcritas. Após a leitura das transcrições, realizou-se o agrupamento das respostas por similaridade para elaboração das categorias para análise. A análise dos dados seguiu a técnica de análise de dados de conteúdo proposto por Bardin 5 .

A pesquisa atendeu aos princípios éticos e científicos conforme a Resolução do CONEP ${ }^{\circ}$ 466 de 2012. O projeto de pesquisa foi submetido e aprovado pelo Comitê de Ética em Pesquisa CEP FAMEBLU.

\section{Resultados e Discussão}

As entrevistas foram realizadas com dez idosos, destes cinco homens e cinco mulheres, com idade entre 66 a 77 anos. Após a transcrição e a tabulação dos dados, foram relacionadas as seguintes informações pessoais, conforme tabela 1.

A maioria dos participantes $(70 \%)$ reside com seus familiares e os demais $(30 \%)$ moram sozinhos. Dos dez entrevistados, quatro são casados, quatro divorciados, um solteiro e um viúvo, formando um grupo bastante heterogêneo. Destes, $60 \%$ mantém vida sexual ativa. Dos $40 \%$ que não tem atividade sexual, apenas $10 \%$, afirmou ser uma opção pessoal por não ter mais vontade de relacionar-se sexualmente. Desta forma, $90 \%$ dos entrevistados mantêm seu desejo sexual ativo. 
Tabela 1. Características dos idosos entrevistados.

\begin{tabular}{|c|c|c|c|c|c|}
\hline Entrevistado & Idade & Com quem vive & Estado civil & $\begin{array}{c}\text { Vida sexual } \\
\text { ativa }\end{array}$ & $\begin{array}{c}\text { Uso de preserva- } \\
\text { tivo }\end{array}$ \\
\hline M1 & 71 & Familiares & Divorciada & Sim & Sim \\
\hline H1 & 68 & Sozinho & Divorciado & Sim & Raramente \\
\hline M2 & 70 & Familiares & Casada & Sim & Não \\
\hline H2 & 74 & Familiares & Viúvo & Não & Não \\
\hline H3 & 73 & Familiares & Casado & Sim & Raramente \\
\hline M3 & 66 & Familiares & Casada & Sim & Não \\
\hline M4 & 77 & Sozinha & Divorciada & Não & Não \\
\hline M5 & 67 & Sozinha & Divorciada & Não & Sim \\
\hline H4 & 69 & Familiares & Solteiro & Não & Sim \\
\hline H5 & 66 & Familiares & Casado & Sim & Sim \\
\hline
\end{tabular}

Fonte: Dados da Pesquisa, 2016.

A partir das entrevistas realizadas e os resultados obtidos após a análise, elencaram-se três categorias de análise: sexualidade na terceira idade: os aspectos físicos e emocionais sob o olhar dos idosos; a percepção dos idosos sobre as infecções sexualmente transmissíveis; a visão do idoso e o seu entendimento sobre a prevenção de ist.

\section{Sexualidade na Terceira Idade: os Aspectos} Físicos e Emocionais sob o Olhar dos Idosos

Em relação ao comportamento sexual e a idade avançada, sabe-se que existem várias alterações fisiológicas e emocionais, que podem dar início ao declínio das atividades sexuais, na qual as pessoas com mais de 60 anos passam a ver a vida em sua plenitude de existência e não apenas esperando a morte chegar, veem também a redescoberta da extensão do interesse sexual, quebrando esse tabu de que idosos não podem praticar o sexo ${ }^{6}$.

Os entrevistados referiram que a terceira idade vem acompanhada por mudanças fisiológicas e psicológicas entre homens e mulheres, conforme relato do entrevistado:

“[...] Perdi mais sim, mudanças no corpo físico e aspectos emocionais, a gente vai envelhecendo (M6).

A idade avançada acentua problemas de saúde e neurológicos que, no entanto, podem afetar a vida sexual nesta faixa etária. Com o aumento da população idosa, a sexualidade na maturidade vem gerando um interesse científico. Sabe-se que a idade não dessexualiza o indivíduo, o que existe, na verdade, são apenas modificações quantitativas da resposta sexual, ou seja, a vida sexual transforma-se constantemente ao longo de toda a evolução individual, porém, só desaparece com a morte ${ }^{7}$.

"[...] Depois de certa idade, a gente não é igual jovem e tem muitas coisas que influenciam na parte sexual, uma é o trabalho, mulher cansada; existe um certo respeito de um e de outro, diferente de quando é jovem" (H5).

Envelhecer não deve significar, necessariamente, declínio ou perda das faculdades e funções ${ }^{7-8}$. A sexualidade apesar de não existir como quando eram jovens em termos de frequências $e$ de qualidade, ela sempre será um benefício ao ato 
praticado. A mudança no comportamento sexual é determinada por fatores biológicos, psicológicos e afetivos. Se os cônjuges mantêm uma compatibilidade e interesses mútuos ao longo de suas vidas, as relações sexuais se manterão presentes ${ }^{9}$. Conforme relato de um dos entrevistados:

"[...] Nós dois nunca brigamos, temos o gosto de ficar um perto do outro, ele já é mais ativo que eu, mesmo sendo mais velho que eu, eu ainda tenho relação porque ele é um ótimo marido" (M6). "[...] Sim houve mudanças, o interesse sexual diminui, sei pelo meu geriatra, que o meu hormônio testosterona está em baixa, tenho que fazer reposição hormonal, para conseguir uma melhora, não ficar nervoso, ansioso" (H9).

A fisiologia sexual não ocorre de forma uniforme entre todos os homens, porém, as alterações mais comuns são caracterizadas por aspectos como: ereção mais flácida, sendo necessário mais tempo para alcançar o orgasmo; ereções involuntárias noturnas diminuem; ejaculação retardada e redução do líquido pré-ejaculatório ${ }^{10}$. Entre mulheres, as alterações se iniciam na menopausa com a diminuição dos hormônios, a pele tende a ficar mais fina e seca, a lubrificação vaginal diminui, levando ao orgasmo em menor duração ${ }^{11}$.

O envelhecimento naturalmente leva a algumas alterações na resposta aos estímulos sexuais, sendo compreendido quando entendemos que as doenças que favorecem o aparecimento das disfunções ${ }^{9}$. Observou-se nas entrevistas que alguns idosos acreditam que diante das alterações fisiológicas decorrente do envelhecimento, existe um declínio da prática, mas não deixam de se adaptar a idade para dar continuidade à vida sexual.

"[...] Um pouco de mudança sempre existe porque a idade chega, já ficamos mais fracos, a cabeça muda um pouco, ficamos mais tranquilos em relação a isso" (H10).

$\mathrm{Na}$ realidade, o idoso continua tendo impulso e atividade sexual, embora ocorram alterações, devido às mudanças fisiológicas, expectativas socioculturais, problemas de saúde e medicações $^{12}$. Os resultados da pesquisa evidenciaram que os idosos ainda praticam a atividade sexual, até mesmo como um gesto de carinho e respeito aos seus parceiros, porém com a frequência da atividade sexual diminuída.

"[...] Diferente de quando é jovem, às vezes não é o fato do ato sexual em si, ir lá e fazer a penetração. Às vezes é o certo carinho, o abraço, a conversa. Então, uma certa idade tem uma porção de coisa que gira, a gente numa certa idade começa a entender, compreender e o certo respeito" (H5).

A sexualidade cria desenvolturas evolutivas que vão desde o nascimento até a morte, evidenciando o desejo sexual que permanece intacto e a persistência da vontade de intimidade e afetividade, tão reprimida na velhice ${ }^{13}$.

\section{A Percepção dos Idosos sobre as Infecções Sexualmente Transmissíveis}

Observam-se mudanças comportamentais na área de sexualidade dos idosos. Com o avanço de técnicas médicas para melhorar disfunções sexuais, terapias orais para disfunção erétil e renovações na reposição hormonal, que contribuíram para o desempenho sexual na qualidade e na frequência das relações sexuais ${ }^{14}$. Alguns dos entrevistados da pesquisa afirmaram que mesmo com certas dificuldades, não deixaram de realizar suas atividades sexuais e expressaram suas concepções sobre as IST.

"[...] Eu me separei com 36 anos e tive outros amores, e nunca peguei uma doença infecciosa, sempre me 
cuidei, sendo solteira e se cuidar nessa idade, você pode ter muitos amores" (M1).

Quando questionados sobre as infecções sexualmente transmissíveis, todos os idosos responderam que sabiam o que eram, mas que não lembravam os nomes das IST. As que foram lembradas por eles foram: HIV, Aids, sífilis e gonorreia. Os idosos evidenciaram que essas infeções são contagiosas, e mencionaram que acontece durante $o$ ato sexual.

"[...] Já ouvi falar, no momento não me lembro do nome das doenças, mas tem a infecções que elas são contagiosas, que passam durante o sexo do parceiro para a parceira" (M8).

Em contrapartida, certos participantes mencionaram que não corriam risco de se contaminarem, pelo fato de serem casadas e terem o contato com um único homem, outros por confiarem na relação segura:

“[...] Hoje tem a AIDS, antigamente no meu tempo, tinha gonorreia, meu marido foi meu único marido e não espero ter outro nesta idade" (M3). "[...] Nunca me preocupei, ele também nunca teve relação com outra mulher, nunca saímos distante um do outro e ele diz que sou a mulher mais linda do mundo e a única dele" (M6).

Outros sujeitos confirmaram que nunca levaram uma vida sem falta de respeito com suas esposas, mesmo já tendo relações anteriormente com outros parceiros.

"[...] Eu graças a Deus nunca tive problema com doenças venéreas, nunca tive porque nunca levei uma vida de boemia, por mais que a gente tinha muita amizade, a gente sempre pro- curava escolher com quem a gente saia, para evitar coisas" (H5).

Ao longo da última década, o conhecimento sobre o HIVIAIDS vem aumentado. Apesar disso, não se pode notar semelhante tendência em relação a outras doenças sexualmente transmissíveis $^{15}$. O que se mostra preocupante, pois mesmo sabendo dos riscos que correm, esses idosos, nem sempre usam camisinha, por acharem não ser necessário ou por confiarem na relação de ambos. Porém, outros idosos entrevistados acreditam na importância de preservação e afirmam que sempre irão usar métodos preservativos.

"[...] Nunca peguei uma doença infecciosa, sempre me cuidei". [...] A pessoa que tem que ver mesmo para se prevenir sempre, os jovens, o povo da rua eu dizia para eles se cuidarem, para não pegarem doenças transmissíveis" (M1).

[...] Já usei preservativo e se fosse para ter relação eu usaria" (M8).

Somados a isto, existe grande resistência por parte dos idosos em usar preservativos, pois existe uma falsa impressão da inutilidade dele na vida sexual, visto que as mulheres com mais de 60 anos não podem engravidar ${ }^{16}$. Mas, conforme relatos da pesquisa, tanto o homem quanto a muIher deixam de usar preservativo por confiarem no parceiro.

"[...] Já usei camisinha quando tive dúvidas das pessoas" $(\mathrm{H} 2)$.

"[...] Nunca usei preservativo, ele e eu sempre vamos ao médico, cuidamos direitinho" (M3).

"[...] Nunca usei nada, nunca me preocupei, ele também nunca teve relação com outra mulher" (M6).

Segundo a Portaria $n^{\circ}$ - 204/2016 do Ministério da Saúde, as informações concentram-se em HIVIAIDS e Sífilis. Atualmente, na literatura existem 
poucos trabalhos sobre prevenção as infecções sexualmente transmissíveis em idosos, sendo um entrave na mensuração do conhecimento dessa faixa etária sobre o assunto.

\section{A Visão do Idoso e o Seu Entendimento sobre a Prevenção de Infecções Sexualmente Trans- missíveis}

O grande desafio da assistência em saúde consiste em prevenir o aumento do número de idosos contaminados, assegurando uma longevidade bem-sucedida. Para isso, na prevenção das IST em idosos, se vê a necessidade em educação em saúde na abordagem deste assunto. A educação em saúde para os idosos sobre essas doenças e a sexualidade podem ser abordadas na terceira idade para constituir um meio de prevenção e promoção de doenças, visto que os profissionais de saúde por meio do conhecimento cientifico, adotam em suas vidas o processo de novos hábitos e condutas de saúde ${ }^{17}$.

Com relação às estratégias de prevenção às IST na terceira idade, evidenciou-se a importância que vários participantes dão ao uso da prevenção, conforme relatos:

"[...] Eu acho que na verdade a vida toda o homem e a mulher tem que se cuidar, tive a relação sexual quando casei" (M3).

"[...] Sim, tem muitas pessoas que não sabem e acabam se contaminando, por falta de conhecimento" $(\mathrm{H} 4)$.

Conforme alguns relatos evidenciados, a importância para prevenção das IST, ocorre devido às mudanças fisiológicas do processo de enveIhecimento, pela queda da imunidade, estando suscetíveis a um tratamento mais complicado, por acharem que perderam sua alegria de vida, e até mesmo por ficarem sozinhos:

"[...] Sim, se chegar de acontecer de pegar uma doença, como você vai se valer, se os de casa não te cuidam quando esta sã, me diga depois que estiver na cama e o mal-estar que dá na mulher, que não pode fazer mais nada, nem se divertir por que está contaminada" (M1).

"[...] É importante porque a nossa defesa do organismo está geralmente em baixa, então eu acredito que a pessoa na terceira idade corre um risco maior" (H9).

"[...] Sim, a idade que nós temos, se nós não nos prevenirmos, vocês sabem melhor que eu, que logo vai para o cemitério, já está com a imunidade mais fraca" (H10).

Os idosos citados apresentam uma certa noção de autocuidado, ou, mais propriamente, auto atenção, em razão da natureza preventiva de suas preocupações, considerando a fragilidade de sua saúde em razão da idade, o que os induz a terem certas atitudes no sentido de minimizar riscos ${ }^{18}$.

As estatísticas referentes às doenças ligadas à vida sexual na terceira idade têm demonstrado que seu crescimento é, consideravelmente, alto em nível mundial e que, estes estão camuflados por conta de um fator retrógrado, mas que permanece bastante ativo: o preconceito e a falta de informação, o que vem contribuindo para tal alarmante crescimento. Acredita-se que as IST estão em todas as classes sociais, tanto os de maior escolaridade quanto os menos instruídos, percebendo a maior vulnerabilidade entre os idosos, sendo confirmado que o número de casos de AIDS na terceira idade no Brasil, sendo a maior percursora de infectados, cresceu como em nenhuma outra faixa etária, ultrapassando o número de casos da epidemia entre os adolescentes de 15 a 19 anos $^{19}$.

O aumento da população idosa no mundo permite o surgimento de novas vulnerabilidades em relação às IST. Neste contexto, os idosos devem ter mais acesso à saúde e à informação de forma a evitar os comportamentos de risco. Nessa concepção, a educação em saúde é definida como um processo educativo de construção de conhecimen- 
tos em saúde, que contribuam para autonomia da população e a inclusão social na participação do seu cuidado em relação à saúde ${ }^{20,21}$.

\section{Conclusão}

O estudo permitiu constatar que os indivíduos mantêm atividade sexual na terceira idade, pois sentem desejo sexual, ainda que a conotação do relacionamento sexual neste grupo não seja necessariamente idêntica ao de pessoas mais jovens. Os idosos possuem plena consciência das transformações psicobiológicas ocorridas em seu organismo, em decorrência do envelhecimento, que podem diminuir a frequência do ato sexual, mas não a capacidade de sentir prazer, os resultados são similares aos encontrados em outros estudos nacionais ${ }^{22,23}$.

Observou-se que os entrevistados demonstraram conhecimento sobre as IST e o método preventivo. Porém, por razões diversas, optam,

\section{REFERÊNCIAS}

1. MASCHIO MBM, et al. Sexualidade na terceira idade: medidas de prevenção para doenças sexualmente transmissíveis e AIDS. Rev. Gaúcha Enferm. 2011; 32(3): 583-9.

2. SANTOS LV, et al. Infecções sexualmente transmissíveis em idosos: revisão sistemática de literatura. Congresso Internacional de Envelhecimento Humano, Campina Grande PB. Brasil. 2013.

3. BRASIL, Secretaria de Atenção à Saúde. Departamento de Atenção Básica. Envelhecimento e saúde da pessoa idosa. Ministério da Saúde, Secretaria de Atenção à Saúde, Departamento de Atenção Básica. Brasília: Ministério da Saúde, 2006.

4. SILVA EMM, et al. Mudanças Fisiológicas e Psicológicas na Velhice Relevantes no tratamento odontológico. Rev. Ciênc. Ext. 2005; 2(1): 74.

5. BARDIN, Laurence. Análise de conteúdo. Lisboa: Edições 70, 2009. Disponível em: <http://pt.slideshare.net/alasiasantos/analise-de-conteudo-laurence-bardin>Acesso em 13 nov. 2016.

6. SOUSA JL. Sexualidade na terceira na terceira idade: uma discussão da aids, envelhecimento e medicamentos para disfunção erétil DST - J Bras Doenças Sex Transm. 2008; 20(1): 59-64 em sua maioria, pelo não uso dos preservativos masculinos e/ou femininos, restringindo seu uso a finalidades contraceptivas e a situações em que identificam a vulnerabilidade de algum risco à saúde.

Esta negligência parece um contrassenso, visto que em sua maioria, os idosos demonstraram conhecimento sobre a presença das doenças. Compreende-se que esse público pertence a um grupo vulnerável, que exige uma equipe de saúde preparada e qualificada para abordagem de sua sexualidade e comportamento correlato.

Os profissionais de saúde devem esclarecer as dúvidas, criando uma relação confiável para um melhor diálogo por meio de uma abordagem facilitadora ao trabalho da equipe de saúde, garantindo a prática saudável do sexo, exigindo uma atenção especial por se tratar de pessoas com características peculiares, que acabam deixando de buscar informações por medo de crítica da família e da sociedade.

7. ALENCAR DL, et al. Fatores que interferem na sexualidade de idosos: uma revisão integrativa. Ciência \& Saúde Coletiva. 2014; 19: 3533-42.

8. TERRANL. Envelhecendo com qualidade de vida: Programa Geron da PUCRS. 2. reimp. Porto Alegre: EDIPUCRS, 2002. 203p.

9. FAJARDO RS, et al. Apostila Sábio e Saudável: uma nova visão da 3 a idade. Araçatuba: FAPESP, 2003. 91p.

10. JUSTO $D$ et al. Sexual activity and erectile dysfunction in ederly men with angiographically documented coronary artery disease. Int J Impot Res. 2010; 22(1):40-44.

11. GRANDIM C.V.C.; SOUSA A.M.M; LOBO J.M. A prática sexual e o envelhecimento. Cogitare enferm. 2007; 12(2):204-213.

12. LEITEA MT, MOURA C, BERLEZI EM. Doenças sexualmente transmissíveis e HIVIAIDS na opinião de idosos que participam de grupos de terceira idade. Bras Geriatr Gerontol. 2007;10(3):339-54.

13. GRADIM CVC, SOUSA AMM, LOBO JM. A prática sexual e o envelhecimento. Cogitare Enfermagem. 2007; 12(2):204-13

14. COCHRAN W. Sampling Techniques. 3 rd ed., New York: John Wiley \& Sons, 1986. 
15. VERAS RP, CAMARGO J K. Idosos e universidade: parceria para qualidade de vida. In. Veras, R. (org.) Terceira idade: um envelhecimento digno para o cidadão do futuro. Rio de Janeiro: Relume-Dumará - UnATI - UERJ 1995, p. 11-27

16. FRICKE K. Pilot Project to introduce simple technical procedures for mechanical biological treatment of waste in Brazil, 2004. Disponível em: <http://www.geocities.ws/ xvi.comau/anais/trabalhos/matsuoka.pdf> Acesso em: 18 de nov. 2016.

17. ALVES, Vânia Sampaio. Um modelo de educação em saúde para o programa saúde da família: pela integralidade da atenção e reorientação do modelo assistencial. Interface. Comunicação em Saúde. 2005; 9(16):39-52.

18. MENENDEZ EL. Modelos de atención de los padecimientos de exclusiones teóricas y articulaciones prácticas. Ciênc. Saúde Coletiva. 2003; 8(1): 185-207.

19. BRASIL, DATA SUS. Casos de AIDS identificados no Brasil, 2009. Disponível em: <http://www.aids.gov.br/cgi/ deftohtm.exe?tabnet/br.def> Acesso em 09 nov. 2016.

20. ROBALO MJ. Implementação e avaliação de um programa de prevenção das IST no idoso. DGES, nº 6037 / 2007 publicado em Diário da Republica $2^{\mathrm{a}}$ série de 23 de março, 2007.
21. PINAFO E et al. Relações entre concepções e práticas de educação em saúde na visão de uma equipe de saúde da família. Trab. Educ. Saúde. 2011, 9(2):201-221.

22. BESSA MEP, et al. Percepção de idosos residentes em instituições de longa permanência acerca da sexualidade na terceira idade. Escola de Saúde Pública do Ceará v.4.n.2. 2010

23. SANTANA MAS, et al. Sexualidade na terceira idade: compreensão e percepção do idoso, família e sociedade. Revista da Universidade Vale do Rio Verde, Três Corações. 2014; 12(1):317-326.

\section{CORRESPONDÊNCIA}

Laís Carolini Theis

Rua Romédio Dorigo, 85 apto 1203 bloco B.

Bairro: Água Verde - Curitiba/PR

CEP: 80.620-140

E-mail: laistheis@gmail.com 